\title{
Phase transitions in simple fluids: An application of a one-phase entropic criterion to Lennard-Jones and point Yukawa fluids
}

\author{
E. Lomba and J. L. López-Martín \\ Instituto de Química Física Rocasolano, Consejo Superior de Investigaciones Científicas, Serrano 119, E-28006 Madrid, Spain \\ and Departamento Química Física I, Universidad Complutense, E-28040 Madrid, Spain \\ H. M. Cataldo* and C. F. Tejero \\ Facultad de Ciencias Físicas, Universidad Complutense, E-28040, Madrid, Spain
}

(Received 20 December 1993; revised manuscript received 14 February 1994)

\begin{abstract}
A recently proposed entropic criterion [P.V. Giaquinta and G. Guinta, Physica A 187, 145 (1992)] for the determination of phase transitions in simple fluids is applied to two-fluid models, a purely repulsive point Yukawa fluid, and a 6-12 Lennard-Jones system. Both the gas-liquid and the freezing transitions are investigated by means of integral equation theory, and assessed with simulation data available in the literature. Our results indicate that the entropic criterion is a reasonable tool for predicting the freezing transition at low temperatures, in particular for purely repulsive potentials. Comparison with other melting rules is less favorable when there is an important attractive component in the interaction. On the other hand, the determination of the gas-liquid critical point and the liquid side of the gas-liquid coexistence curve is merely qualitative. Our results, however, show the existence of a correlation between the gas-liquid transition and the location of one of the inflection points of the density-dependent excess residual entropy.
\end{abstract}

PACS number(s): 61.20.Gy, 64.70.-p

\section{INTRODUCTION}

The use of integral equation techniques for the determination of phase diagrams in simple fluids has been widely explored [1-3]. In particular, a new suggestive criterion that enables the determination of the melting line with one-phase thermodynamic and structural properties as input has been recently proposed by Giaquinta and Giunta [4]. They made a careful analysis of the density dependence of the excess entropy $s^{\mathbf{e x}}$ and the pair entropy $s_{2}$ (i.e., the contribution of spatial correlations between pairs of particles to the excess entropy) for the hard-sphere fluid, and concluded that the residual entropy $\Delta s(\rho)=s^{\text {ex }}-s_{2}$ reflects all the borders between structural and dynamical regimes present in the fluid, through the zeros of the function itself and its derivatives [4]. This somewhat empirical criterion was successfully tested in the prediction of the melting line for the hardsphere fluid [4], the Lennard-Jones (LJ) fluid [5], and in a very recent work for a system of rigid $\mathrm{C}_{60}$ molecules [2].

In this paper we intend to explore this one-phase criterion for a system with attractive interactions, the 6$12 \mathrm{LJ}$ fluid, along the full density range, analyzing not only the melting transition, but also the correlations between inflection points and the liquid-vapor transition. We also consider a purely repulsive soft potential, the

\footnotetext{
*Permanent address: Departamento de Física, Facultad de Ciencias Exactas y Naturales, Universidad de Buenos Aires, 1428 Buenos Aires, Argentina.
}

point Yukawa, for which the location of the freezing transition has been the focus of recent work [6]. This interaction, though simple, has been successfully applied to model colloidal suspensions [7], which are known to undergo transitions between dense disordered and dense ordered phases [8].

Spatial correlations and thermodynamics have been evaluated by means of the reference hypernetted chain (RHNC) equation [9], a theoretical approach that has proved extremely accurate for simple fluids. We have solved the RHNC equation for a 6-12 LJ system for which the interaction reads

$$
\beta u_{\mathrm{LJ}}(r)=\frac{4 \epsilon}{k_{B} T}\left[\left(\frac{\sigma}{r}\right)^{12}-\left(\frac{\sigma}{r}\right)^{6}\right],
$$

with $\epsilon, \sigma$ being the Lennard-Jones parameters and $\beta=$ $1 / k_{B} T$ the inverse temperature; and also for a repulsive point Yukawa fluid, in which one has

$$
\beta u_{Y}(r)=\frac{\epsilon_{Y}}{k_{B} T} \frac{\sigma_{Y}}{r} \exp \left[-\frac{r}{\sigma_{Y}}\right] .
$$

In this case it is usual [6] to introduce the reduced parameters $t=1 / \beta \epsilon_{Y}$ and $\lambda=a / \sigma_{Y}$, with $a$ the average nearest-neighbor distance, i.e., $\rho=1 / a^{3}, \rho$ being the average number density. Thus, in units of length $a$, the interaction reads

$$
\beta u_{Y}(r)=\frac{1}{t \lambda r} \exp (-\lambda r) .
$$

For these interaction potentials, the Ornstein-Zernike (OZ) equation 


$$
h(r)=c(r)+\rho \int c\left(r_{13}\right) h\left(r_{32}\right) d \mathbf{r}_{3}
$$

has been solved using standard numerical techniques [1]. In the expression above $h(r)$ is the total correlation function and $c(r)$ is the direct correlation function. This relation is coupled with the RHNC closure, which reads

$$
h(r)=\exp \left[-\beta u(r)+h(r)-c(r)+b_{0}(r)\right]-1 .
$$

Here, the reference system bridge function $b_{0}(r)$ is computed for a hard-sphere fluid using the Verlet-WeisHenderson-Grundke parametrization $[10,11]$, and the hard-sphere diameter is determined by requiring the minimization of the free energy defined in Ref. [9]. All the thermodynamic quantities can be evaluated using the RHNC thermodynamics [see Eqs. (11) and (22)-(23) in Ref. [9]]. With these, we can compute the excess entropy per particle (in units of Boltzmann's constant $k_{B}$ )

$$
s^{\mathbf{e x}}=\beta\left(u^{\mathbf{e x}}-a^{\mathbf{e x}}\right)
$$

from the excess internal energy per particle, $u^{\text {ex }}$, and the excess free energy per particle, $a^{\text {ex }}$. The two-particle configurational contribution to the excess entropy is given by $[4,12]$

$$
s_{2}=-\frac{1}{2} \rho \int g(r) \ln g(r) d \mathbf{r}+\frac{1}{2} \rho \int[g(r)-1] d \mathbf{r}
$$

with $g(r)$ the pair distribution function. Hence we can analyze in a straightforward way the density dependence of $\Delta s(\rho)$ and study its zeros, extrema, and inflection points. Numerically, it will not be feasible to go beyond the second derivative, since truncation errors along the numerical solution of the integral equation are enhanced by the differentiation.

\section{RESULTS}

Our calculations for the LJ system are collected in Tables I-III, and Figs. 1 and 2. In Table I we compare our results for $\beta u^{\text {ex }}$, the compressibility factor $\beta P / \rho, s^{\text {ex }}$, and $s_{2}$, with those of an extensive Monte Carlo (MC) calculation by Giaquinta et al. [5]. We observe that, despite the accuracy of the RHNC thermodynamics, the zeros of the residual entropy $\Delta s(\rho)$ are somewhat shifted toward higher densities. The largest source of the error in the RHNC approximation stems from the evaluation of $s_{2}$, a quantity which is affected by large relative errors, even at $T^{*}=1.15$, while the remaining properties are accurately described by the RHNC. An alternative integral equation approach, the self-consistent hybrid mean spherical approximation (HMSA), previously investigated by Caccamo, Giaquinta, and Giunta [3], yields somewhat better results, in particular at high temperatures (see Table III); nonetheless, the RHNC approximation has the advantage that it does not require thermodynamic integration, since this approach is endowed with explicit expressions to determine the thermodynamic potentials directly from the correlation functions.

In previous works $[3,4]$, the existence of a pronounced correlation between the zeros of $\Delta s(\rho)$ and the freezing transition was unequivocally established. It was also suggested that the extrema in $d \Delta s(\rho) / d \rho$ [i.e., zeros of $d^{2} \Delta s(\rho) / d \rho^{2}$ or inflection points in $\left.\Delta s(\rho)\right]$ are associated with the gas-liquid transition. Therefore, we have here investigated the behavior of $d \Delta s(\rho) / d \rho$ for a wide range of temperatures and densities, as can be seen in Fig. 1. Due to the presence of the spinodal line, at the lowest $T^{*}$ no RHNC solution is available for some densities [1]. In Fig. 1, we first observe the presence of two extrema on the high density side, with one of them disappearing for $T^{*}>T_{c}^{*^{\prime}}$ (and this $T_{c}^{*^{\prime}} \approx 1.45$ is somewhat off the simulated critical temperature $T_{c}^{*} \approx 1.30$ [13], or the value extrapolated from the RHNC phase diagram, $T_{c}^{*} \approx 1.31$

\begin{tabular}{|c|c|c|c|c|c|c|c|c|c|c|c|}
\hline \multirow[b]{2}{*}{$T^{*}$} & \multirow[b]{2}{*}{$\rho^{*}$} & \multicolumn{2}{|c|}{$\beta P / \rho$} & \multicolumn{2}{|c|}{$\beta u^{\text {ex }}$} & \multicolumn{2}{|c|}{$s^{e x}$} & \multicolumn{2}{|c|}{$s_{2}$} & \multicolumn{2}{|c|}{$\Delta s$} \\
\hline & & MD & RHNC & $\mathrm{MD}$ & RHNC & MD & RHNC & MD & RHNC & MD & RHNC \\
\hline & 0.84 & 0.441 & 0.449 & -8.032 & -8.028 & -3.441 & -3.514 & -3.378 & -3.306 & -0.063 & -0.208 \\
\hline & 0.85 & 0.692 & 0.705 & -8.112 & -8.109 & -3.516 & -3.590 & -3.492 & -3.406 & -0.024 & -0.184 \\
\hline & 0.86 & 0.952 & 0.977 & -8.179 & -8.188 & -3.581 & -3.667 & -3.599 & -3.509 & 0.018 & -0.158 \\
\hline \multirow[t]{8}{*}{0.75} & 0.87 & 1.244 & 1.268 & -8.256 & -8.265 & -3.659 & -3.746 & -3.711 & -3.617 & 0.052 & -0.129 \\
\hline & 0.88 & & 1.579 & & -8.339 & & -3.284 & & -3.727 & & -0.097 \\
\hline & 0.89 & & 1.906 & & -8.412 & & -3.905 & & -3.842 & & -0.063 \\
\hline & 0.90 & & 2.252 & & -8.481 & & -3.986 & & -3.962 & & -0.024 \\
\hline & 0.91 & & 2.617 & & -8.547 & & -4.068 & & -4.085 & & 0.017 \\
\hline & 0.93 & 5.022 & 5.041 & -5.205 & -5.202 & -3.671 & -3.671 & -3.566 & -3.494 & -0.105 & -0.177 \\
\hline & 0.94 & 5.364 & 5.371 & -5.229 & -5.228 & -3.739 & -3.742 & -3.668 & -3.592 & -0.071 & -0.150 \\
\hline & 0.95 & 5.711 & 5.716 & -5.251 & -5.250 & -3.810 & -3.812 & -3.771 & -3.692 & -0.039 & -0.120 \\
\hline \multirow[t]{4}{*}{1.15} & 0.96 & 6.069 & 6.066 & -5.272 & -5.273 & -3.882 & -3.886 & -3.882 & -3.799 & 0.000 & -0.087 \\
\hline & 0.97 & 6.450 & 6.444 & -5.289 & -5.290 & -3.953 & -3.957 & -3.993 & -3.905 & 0.040 & -0.052 \\
\hline & 0.98 & & 6.835 & & -5.305 & & -4.035 & & -4.015 & & -0.020 \\
\hline & 0.99 & & 7.240 & & -5.318 & & -4.111 & & -4.129 & & 0.018 \\
\hline
\end{tabular}
[1]). We notice that the locus of the maxima in $d \Delta s / d \rho$

TABLE I. Thermodynamics of the 6-12 LJ fluid close to the liquid-solid transition. Theory vs MD results from Ref. [3]. 
TABLE II. Comparison of the locus of maxima in $d \Delta s / d \rho$ (denoted by GG-RHNC) and predictions of the liquid side of the coexistence curve from Gibbs ensemble Monte Carlo (GE-MC), van der Waals theory ( $v W$ ), RHNC chemical equilibrium data (RHNC), and Guggenheim's fit to experimental results $(\mathrm{Gg})$. The data are scaled with the critical point location, i.e., $\rho_{r}^{l}=\rho^{l} / \rho_{c}$ and $T_{r}^{l}=T^{l} / T_{c}$. GE-MC data taken from Ref. [13]. RHNC data taken from Ref. [1]. Gg data taken from Ref. [14].

\begin{tabular}{cccccc}
\hline \hline & \multicolumn{5}{c}{$\rho_{r}^{l}$} \\
$T_{r}$ & GG-RHNC & GE-MC & vW & RHNC & Gg \\
\hline 0.992 & 1.413 & 1.452 & 1.177 & & 1.350 \\
0.954 & 1.484 & 1.629 & 1.442 & 1.594 & 1.660 \\
0.763 & 1.611 & 1.955 & 1.727 & 1.955 & 1.960 \\
0.878 & 1.788 & 2.252 & 2.014 & 2.258 & 2.260 \\
0.687 & 1.897 & 2.429 & 2.164 & 2.439 & 2.423 \\
0.573 & & 2.645 & 2.354 & 2.648 & 2.639 \\
\hline \hline
\end{tabular}

is apparently correlated with the liquid side of the gasliquid transition (it is not possible to say whether it is the spinodal or the binodal line which is correlated), as can be seen in Fig. 2. The prediction of the location of the two-phase boundary is, however, very poor (compare the double-dot-dashed curve with the solid circles from MC simulation). This is seen more explicitly in Table II. Here we compare the location of the maxima in $d \Delta s / d \rho$ (denoted by GG-RHNC) with results from van der Waals theory (vW), Gibbs ensemble MC data from Ref. [13] (GE-MC), results from the RHNC using plain chemical equilibrium conditions [1], and Guggenheim's fit to experimental data [14]. From Fig. 2 we already knew that the critical temperature estimate from the Giaquinta-Giunta rule is quite off both simulated and RHNC predictions. From Table III we find now that the liquid side of the coexistence curve scaled with the critical $T_{c}$ and $\rho_{c}$ is also incorrectly predicted, in particular at low temperatures. Accordingly, one cannot expect to obtain reliable estimates of the gas-liquid transition from the entropic criterion, but just a mere indication that the transition is about to take place.

For the high density minimum in $d \Delta s / d \rho$, we observe that for temperatures approaching from above the pseudocritical $T_{c}^{*^{\prime}}$, it converges toward a value $\rho_{c} \sigma^{3} \approx 0.6$. Giaquinta and Giunta [4] found for the hard-sphere fluid an inflection point in $\Delta s(\rho)$ at $\rho \sigma^{3} \approx 0.5$, which they associated with a transition to a dense fluid regime,

TABLE III. Comparison of the location of the melting line prediction according the Giaquinta-Giunta criterion using RHNC, HMSA, and MD input (GG-RHNC, GG-HMSA, and GG-MD, respectively), the Hansen-Verlet rule with RHNC input (HV-RHNC), and MC simulation. MC data taken from Ref. [15]. GG-HMSA data taken from Ref. [3]. GG-MD data taken from Ref. [5].

\begin{tabular}{cccccc}
\hline \hline & \multicolumn{5}{c}{$\rho^{*}$} \\
\cline { 2 - 5 }$T^{*}$ & MC & GG-RHNC & GG-HMSA & GG-MD & HV-RHNC \\
\hline 0.75 & 0.875 & 0.906 & 0.878 & 0.856 & 0.876 \\
1.15 & 0.936 & 0.985 & 0.950 & 0.960 & 0.944 \\
1.35 & 0.964 & 1.016 & & & 0.973 \\
\hline \hline
\end{tabular}

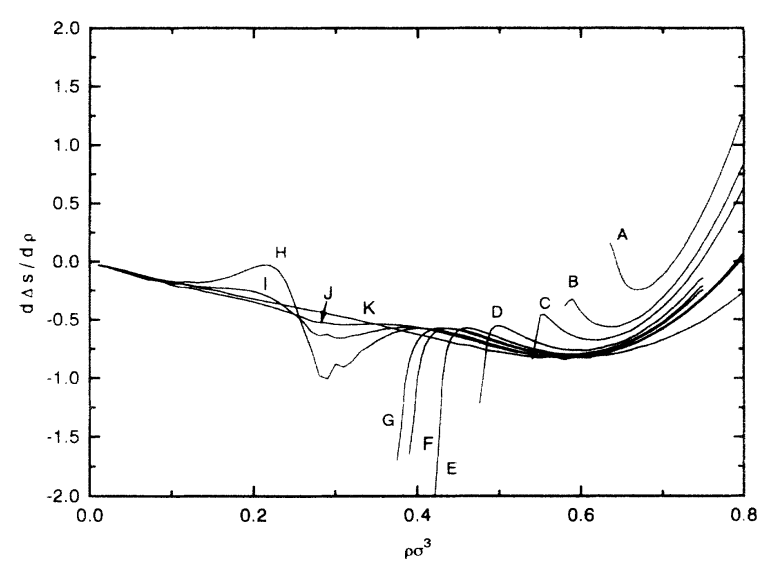

FIG. 1. $d \Delta s / d \rho$ vs $\rho$ for the Lennard-Jones fluid. Curves are labeled according the temperature $T^{*}$ in the following pairs $(A, 0.75),(B, 0.90),(C, 1.00),(D, 1.15),(E, 1.25)$, $(F, 1.30),(G, 1.32),(H, 1.39),(I, 1.42),(J, 1.45)$, and $(K, 2.0)$.

whose boundaries are defined by the percolation threshold. For higher densities the accessible volume in the system breaks into isolated cavities. Since the LJ fluid is more "compressible," it is understandable to find such a transition at a more dense state. For temperatures below the pseudocritical $T_{c}^{*^{\prime}}$, the first order gas-liquid transition interferes with the structural transition and this is shifted toward higher densities, the trend indicating that both inflection points tend to merge. This latter assumption could not be proved since, as can be seen in Fig. 2, at the triple point $T^{*}$, the maximum lies beyond the nonsolution line of the equation.

Focusing now on the solid-liquid transition, we observe in Table III that the Giaquinta-Giunta criterion with RHNC input (GG-RHNC) leads to a freezing line shifted toward higher densities by less than $5 \%$. This

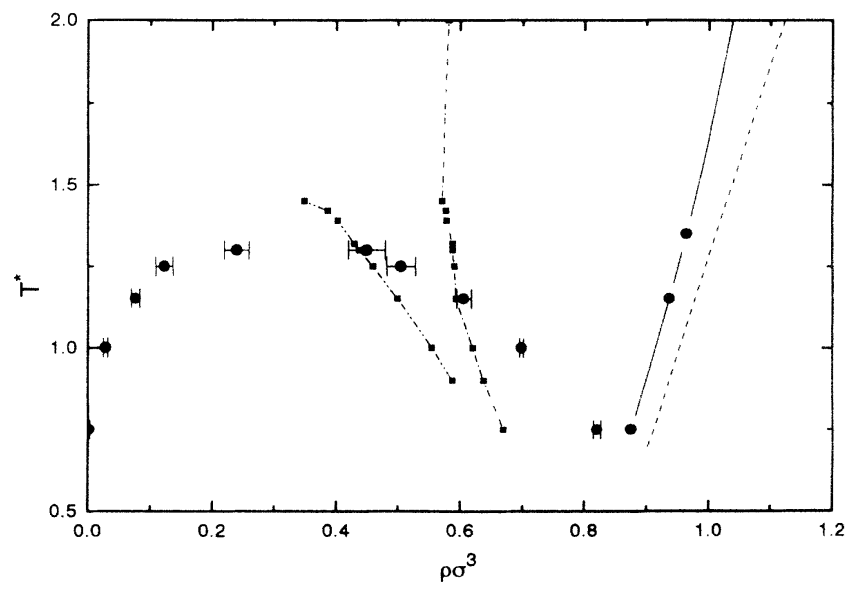

FIG. 2. Phase diagram of the LJ fluid. Filled circles correspond to $\mathrm{MC}$ results from Refs. [13] and [15]. The dashed line is the RHNC prediction of the freezing transition. The dash-dotted line corresponds to the location of the last minimum of $d \Delta s / d \rho$ (estimate of the vapor-liquid transition), and the double-dot-dashed line to the locus of the last maximum (estimate of the percolation threshold). 


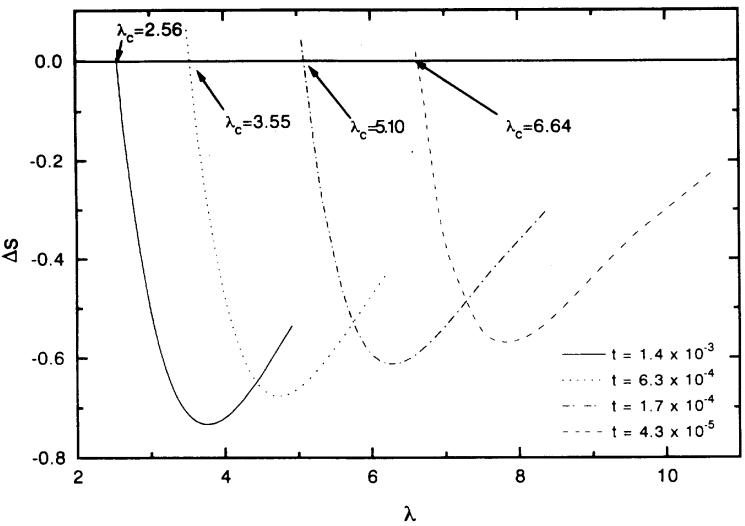

FIG. 3. Residual entropy $\Delta s$ vs $\lambda$, for a repulsive point Yukawa fluid computed in the RHNC approximation.

deviation increases at higher $T$, probably because the transition shows up at larger $\rho$ values where the inaccuracies of the RHNC are enhanced. Use of HMSA or molecular dynamics to provide structural and thermodynamic input improves the results somewhat. Other phenomenological criteria, like the Hansen-Verlet rule (even with RHNC-calculated structure factors, which we here denote as HV-RHNC) or Lindemann's rule $(L=0.14)$, are in reasonable agreement with MC simulations [15]. In particular, HV-RHNC results deviate by less than $1 \%$ from the simulation values. On the whole, the HansenVerlet rule seems more appropriate for the LJ potential, and like the Giaquinta-Giunta criterion, it only requires liquid phase data input.

Concerning the point Yukawa system, we have studied the four temperatures for which Meijer and Frenkel [16] determined the location of the melting transition by MC simulations. Notice that, in this case, the fluid phase can freeze into a body-centered cubic (bcc) or a face-centered cubic (fcc) crystal. Again, RHNC thermodynamics is extremely accurate as compared with MC simulations [16].

In Fig. 3 we show the evolution of $\Delta s(\lambda)$ vs $\lambda$, and our results are explicitly compared in Table IV and Fig. 4 with those of Meijer and Frenkel, who found a bcc (fcc) stable solid structure for the two high (low) temperatures. According to Dupont et al. [17], the fluid-fcc transition at $\lambda=5.33$ corresponds to a metastable fcc crystal whereas for the low-temperature isotherm, the bcc-fcc-fluid triple point is located at $\lambda=6.75$. We see

TABLE IV. Equilibrium density along the liquid-solid coexistence line of the point Yukawa fluid. MC results from Ref. [16] vs Lindemann's rule with MC input (MC-L), Giaquinta-Giunta criterion with RHNC input (GG-RHNC), and the Hansen-Verlet rule with RHNC input (HV-RHNC). MC-L data taken from Ref. [18].

\begin{tabular}{ccccc}
\hline \hline & \multicolumn{3}{c}{$\lambda$} \\
$10^{-4} t$ & MC & MC-L & GG-RHNC & HV-RHNC \\
\cline { 2 - 5 } 14 & $2.94(3)$ & 2.99 & 2.56 & 3.13 \\
6.3 & $3.85(4)$ & 3.86 & 3.55 & 4.05 \\
1.7 & $5.33(5)$ & 5.51 & 5.10 & 5.53 \\
0.43 & $6.77(4)$ & 7.00 & 6.64 & 7.01 \\
\hline \hline
\end{tabular}

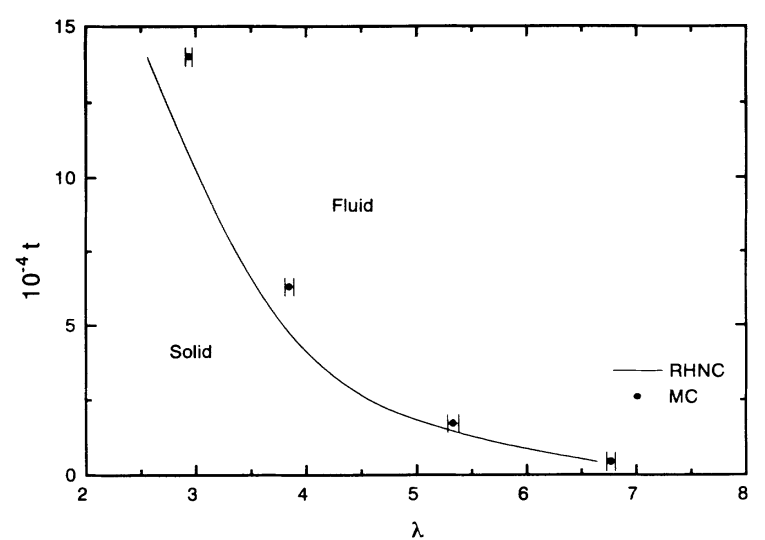

FIG. 4. Liquid-solid coexistence line for the repulsive point Yukawa fluid. RHNC theory vs computer simulation from Ref. [16].

that GG-RHNC results are considerably more accurate than other empirical rules at low temperature, but again deviations increase at high temperature (low $\lambda$, i.e., high densities). The location of the melting transition using Lindemann's rule $(L=0.19)$ has also been included in Table III. As shown by Stevens and Robbins [18], this Lindemann's ratio is nearly the same for both crystal structures in infinite systems. It is seen that GG-RHNC gives better results near the triple point. On the other hand, the evaluation of the peak height from simulation in the liquid structure factor is not accurate enough to determine a precise melting temperature for the Yukawa system [18]. If again one uses RHNC input combined with the Hansen-Verlet rule, the results are not so impressive as in the LJ case. One could speculate that the Giaquinta-Giunta criterion might be more suitable for purely repulsive potentials. However, one must bear in mind that low-temperature repulsive Yukawa fluids look increasingly like hard-sphere systems, and this could be underlying the better agreement found for those systems.

Now a question remains to be addressed: the number of extrema in $d \Delta s / d \rho$. In Fig. 5 we have plotted this quantity vs $\rho$ for the lowest $t$ value. Contrary to what was

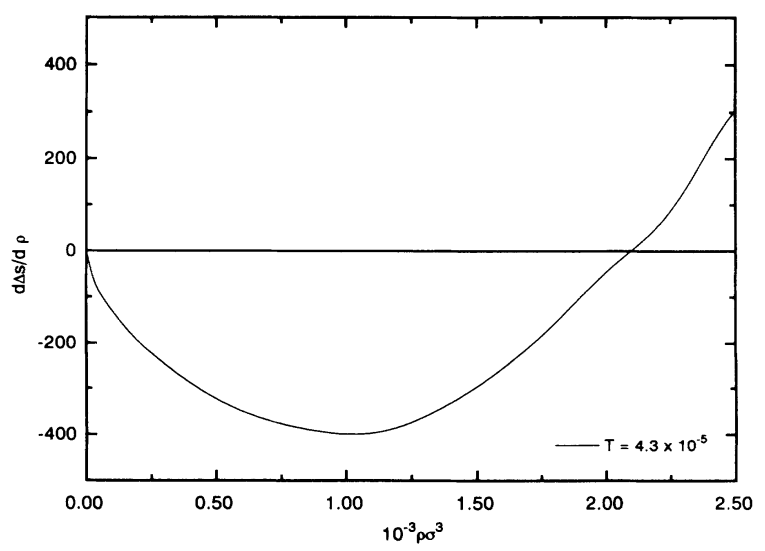

FIG. 5. $d \Delta s / d \rho$ vs $\rho$ for the repulsive Yukawa fluid at $t=4.3 \times 10^{-5}$ computed in the RHNC approximation. 
observed for the low- $T^{*}$ LJ fluid (see Fig. 1) here only one minimum shows up (as in the hard-sphere case [4] or in the $\mathrm{LJ}$ case above $T_{c}^{*^{\prime}}$ ). This minimum can probably be associated with the dense fluid regime transition related to the percolation threshold, as put forward by Giaquinta and Giunta in the hard-sphere fluid case [4]. The absence of the high density maxima found in the LJ fluid is due to the purely repulsive nature of the interaction and the consequent lack of spinodal behavior.

We can conclude that the use of the one-phase entropic criterion appears to be reliable for pure repulsive potentials at low temperatures (hard-sphere-like conditions), but its accuracy is exceeded by the Hansen-Verlet rule in systems with attractive interactions. In this latter case, the Giaquinta-Giunta criterion also gives evidence of the gas-liquid transition, but its location is inconclusive and the criterion cannot match more traditional procedures, such as the simple use of thermodynamic equilibrium conditions with integral equation input. It remains to be assessed what portion of this inaccuracy can be ascribed to the integral equation itself. As to the freezing transition, we know that most discrepancies found in this work are due to the inaccuracy of the RHNC theory in the calculation of $s_{2}[5]$.

\section{ACKNOWLEDGMENTS}

This work has been financed by Spanish Dirección General de Investigación Científica y Técnica (DGICYT) under Grants No. PB91-0110 and PB91-0378.
[1] E. Lomba, Mol. Phys. 68, 87 (1989).

[2] A. Cheng, M. L. Klein, and C. Caccamo, Phys. Rev. Lett. 71, 1200 (1993).

[3] C. Caccamo, P. V. Giaquinta, and G. Giunta, J. Phys. Condens. Matter 5, B75 (1993).

[4] P. V. Giaquinta and G. Giunta, Physica A 187, 145 (1992).

[5] P. V. Giaquinta, G. Giunta, and S. P. Giarritta, Phys. Rev. A 45, R6966 (1992).

[6] C. F. Tejero, J. F. Lutsko, J. L. Colot, and M. Baus, Phys. Rev. A 46, 3373 (1992).

[7] P. Salgi, J. F. Guerin, and R. Rajagopalan, Colloid Polym. Sci. 270, 785 (1992).

[8] M. H. J. Hagen, E. J. Meijer, G. C. A. M. Mooij, D. Frenkel, and H. N. W. Lekkerkerker, Nature 365, 425 (1993).

[9] F. Lado, S. M. Foiles, and N. W. Ashcroft, Phys. Rev. A
28, 2374 (1983).

[10] L. Verlet and J. J. Weis, Phys. Rev. A 5, 939 (1972).

[11] D. Henderson and E. W. Grundke, J. Chem. Phys. 63, 601 (1975).

[12] R. E. Nettleton and H. S. Green, J. Chem. Phys. 29, 1365 (1958).

[13] A. Z. Panagiotopoulos, Mol. Phys. 61, 813 (1987).

[14] L. E. Reichl, A Modern Course in Statistical Physics, 1st ed. (University of Texas, Austin, 1980).

[15] J. P. Hansen and L. Verlet, Phys. Rev. 184, 151 (1969).

[16] E. J. Meijer and D. Frenkel, J. Chem. Phys. 94, 2269 (1991).

[17] G. Dupont, S. Moulinasse, J. P. Ryckaert, and M. Baus, Mol. Phys. 79, 453 (1993).

[18] M. J. Stevens and M. O. Robbins, J. Chem. Phys. 98, 2319 (1993). 